TAO, Vol. 17, No. 1, 111-128, March 2006

\title{
The Formation of Concentric Eyewalls with Heat Sink in a Simple Tropical Cyclone Model
}

\author{
Jia-Yi Peng $^{1,2}$, Ben Jong-Dao Jou ${ }^{1, *}$, Melinda S. Peng ${ }^{3}$, Juan Fang ${ }^{4}$ and Rong-Sheng Wu ${ }^{4}$
}

(Manuscript received 30 November 2003, in final form 29 December 2005)

\begin{abstract}
A linearized, two-layer axisymmetric model analogous to Schubert el al. (1980) is used to simulate the formation of concentric eyewalls in an ideal strong tropical cyclone. By imposing a heat sink near the center of a cyclone the induced perturbation wind, through thermodynamic adjustment to the heat sink, forms a double-peak structure when the disturbance is added to the basic state tangential wind. The heat sink represents, in a crude way, evaporative cooling of precipitation falling from cloud during late stage convective activity or a cooling through environmental advection. Detailed profiling of the induced double-peak wind structure is dependent on the radial profile of the imposed heat sink. After the double-peak tangential wind structure is formed, if a heat source corresponding to a new convective activity is generated inside the outer maximum tangential wind, the outer eyewall contracts and strengthens while the inner eyewall weakens. This result suggests that thermodynamic adjustments to changes in the heating of a tropical-cyclone-core region may contribute to the formation of the double-eyewall phenomenon.
\end{abstract}

(Key words: Tropical cyclone, Concentric eyewall, Simple linear model, Heat sink)

${ }^{1}$ Department of Atmospheric Sciences, National Taiwan University, Taipei, Taiwan, ROC

2 Department of Atmospheric Sciences, Nanjing University of Information Science and Technology, Nanjing, China

${ }^{3}$ Naval Research Laboratory, Monterey, CA

${ }^{4}$ Department of Atmospheric Sciences, Nanjing University, Nanjing, China

* Corresponding author address: Prof. Ben Jong-Dao Jou, Department of Atmospheric Sciences, National Taiwan University, Taipei, Taiwan, ROC;E-mail: jou@hp735.as.ntu.edu.tw 


\section{INTRODUCTION}

Concentric eyewalls have been observed in the life cycle of strong tropical cyclones, those having a minimum sea level pressure below $970 \mathrm{hPa}$ with maximum tangential wind speed greater than $45 \mathrm{~m} \mathrm{~s}^{-1}$ (Chen 1987). Willoughby et al. (1989) identify a double eyewall structure for hurricane Gilbert (estimated minimum sea level pressure of $888 \mathrm{hPa}$ ) with an inner eyewall in the radius of $8-20 \mathrm{~km}$ and an outer eyewall of $55-100 \mathrm{~km}$. A detailed analysis of Gilbert by Black and Willoughby (1992) showed that the primary eyewall appeared first and the outer eyewall formed during the weakening stage of the storm. The outer eyewall strengthened and contracted while the inner eyewall continued to weaken. Finally, the outer eyewall replaced the inner one and completed the so-called eyewall replacement cycle (see Fig.1 for a pictorial description of the cycle).

Studies have been devoted to the understanding of the mechanisms involved in concentric-eyewall formation. Willoughby et al. $(1984,1988)$ suggested that symmetric instability may play an important role in the formation of the outer eyewall. They, however, could not develop a relation between the location of instability and the outer eyewall. Montgomery and Kallenbach (1997) suggested that concentric eyewalls may be the result of radial propagating linear vortex Rossby waves and that there exists a critical radius that limits the propagations. Since the propagation of vortex Rossby waves in a tropical cyclone is a response of the restoring force induced by the radial gradient of the basic state vorticity, the vortex Rossby waves will be confined to a region near the radius of maximum wind (RMW). Nong and Emanuel (2003) discussed the formation of the concentric eyewalls using an axisymmetric model. Their simulations showed that a secondary eyewall may result from a finite-amplitude WISHE instability, triggered by external forcing. Recently, Kuo et al. (2004) demonstrated that concentric eyewalls can be produced as two barotropical vortices of different size and intensity interact under some specified criteria without merging into a monopole. Their results suggest the importance of barotropic dynamics on the formation of concentric eyewalls.

Besides the importance of barotropic dynamics, from Fig.1, it can be seen that the outer eyewall of Gilbert formed during the weakening stage. Shapiro and Willoughby (1982) and Willoughby et al. (1982) used a symmetric model (SW-model) to diagnose the secondary circulation induced by a point heat source in a balanced, axisymmetric vortex. For a heat source near RMW, a maximum of the tangential wind tendency lay just inside the heat source and caused the RMW to propagate inward. SW-model suggested that the inward propagation of the RWM in response to heating provided a plausible physical explanation for the contraction of the outer wind maximum. However, the numerical experiment did not simulate the double-peak structure of the tangential wind that appeared in tropical cyclones with concentric eyewalls.

Schubert et al. (1980) discussed the geostrophic adjustment process in an axisymmetric vortex using a linearized two-layer model. The model consists of equations governing smallamplitude, forced, axisymmetric perturbations on a basic-state of tangential flow that is height independent. When the basic flow is at rest, solutions for the transient and final adjusted state are obtained by Hankel transformations. For the non-resting basic state, they only discuss the final adjusted state without considering the heat source. 


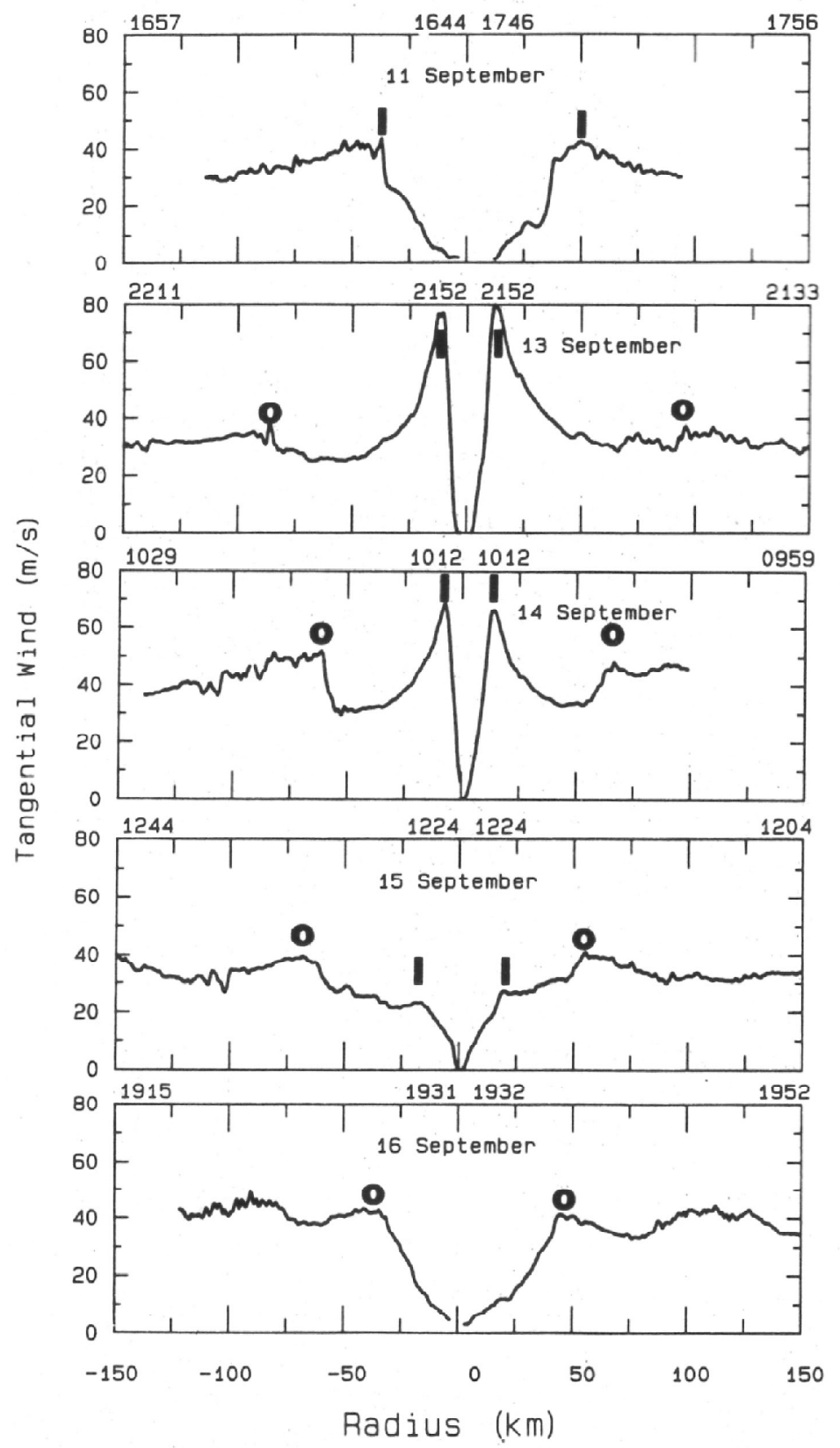

Fig. 1. Flight-level tangential wind speed from traverses through the center of Hurricane Gilbert. Bolds ' $I$ ' and ' $O$ ' denote the location of the inner and outer eyewall wind maximums, respectively. Times at the beginning and end of each radial pass are plotted at the top of the panels (Black and Willoughby 1992). 
The fact that an outer eyewall forms for a strong tropical cyclone during its weakening stage suggests that a cooling of the storm associated with cumulus convection may play a role in the formation of concentric eyewalls. This paper presents that the perturbation in a strong basic state vortex flow, induced by the thermodynamic adjustment process to a heat sink, can form a double-peak feature when the disturbance is imposed on the basic state. The result suggests a possible mechanism contributing to the formation of concentric eyewalls in tropical cyclones.

The paper is outlined as follows. A brief description of the derivation of the governing equations for small-amplitude perturbations and solution methods are given in section 2 . The numerical solutions of the linear perturbations with a heat sink are presented in section 3 . Section 4 discusses the contraction of the outer eyewall with a heat sink. A summary of the paper is given in Section 5.

\section{GOVERNING EQUATIONS AND DIFFERENCE SCHEME}

The formulation of the linear dynamics of the response of an axisymmetric vortex to a heat source is presented below. The framework follows Schubert et al. (1980). By using cylindrical coordinates in the horizontal and pressure in the vertical, the axisymmetric form of the primitive equations can be written as follows:

$$
\begin{aligned}
& \frac{\partial u}{\partial t}+u \frac{\partial u}{\partial r}+\omega \frac{\partial u}{\partial p}-\left(f+\frac{v}{r}\right) v+\frac{\partial \phi}{\partial r}=0, \\
& \frac{\partial v}{\partial t}+u \frac{\partial v}{\partial r}+\omega \frac{\partial v}{\partial p}+\left(f+\frac{v}{r}\right) u=0, \\
& \frac{\partial r u}{r \partial r}+\frac{\partial \omega}{\partial p}=0, \\
& \frac{\partial}{\partial t}\left(-\frac{\partial \phi}{\partial p}\right)+u \frac{\partial}{\partial r}\left(-\frac{\partial \phi}{\partial p}\right)-\sigma \omega=\frac{R}{C_{p} p} Q(r, t),
\end{aligned}
$$

where $u$ is the radial component of velocity, $v$ the tangential component, $\omega$ the vertical $P$ velocity $(\omega=d p / d t), \phi$ the geopotential, $f$ the constant Coriolis parameter [here, $f=5 \times 10^{-5}\left(\mathrm{~s}^{-1}\right)$, and $\sigma$ the static stability defined by $\sigma=-\rho^{-1}(\partial \ln \theta / \partial p)$.

Following Schubert et al. (1980) to simplify the system into a two-layer version, Eqs (1a-d) become, in a nondimensional form:

$$
\frac{\partial u_{d}}{\partial t}-\left(1+\frac{2 \bar{v}}{r}\right) v_{d}+\frac{\partial \phi_{d}}{\partial r}=0
$$




$$
\begin{aligned}
& \frac{\partial v_{d}}{\partial t}+(1+\bar{\zeta}) u_{d}=0, \\
& \frac{\partial \phi_{d}}{\partial t}+\frac{\partial r u_{d}}{r \partial r}=Q(r, t), \\
& \frac{\partial u_{a}}{\partial t}-\left(1+\frac{2 \bar{v}}{r}\right) v_{a}+\frac{\partial \phi_{a}}{\partial r}=0, \\
& \frac{\partial v_{a}}{\partial t}+(1+\bar{\zeta}) u_{a}=0, \\
& \frac{\partial r u_{a}}{r \partial r}=0,
\end{aligned}
$$

where $\bar{\zeta}=\partial r \bar{v} / r \partial r=\bar{v} / r+\partial \bar{v} / \partial r$ is the basic state relative vorticity and subscript "d" denotes the difference between the two layers, one at $250 \mathrm{mb}$ (level 1) and another at $750 \mathrm{mb}$ (level 3) and subscript "a" denotes the sum of the two layers. Eqs (2a - c) are identical to (2.8 - 2.10) in Schubert et al. (1980) except that there is an additional angular momentum source term in their (2.9).

From equation (2f) we know that $r u_{a}$ must be independent of $r$. From the boundary condition:

$$
\begin{aligned}
& r \rightarrow 0, u_{a} \rightarrow 0, \\
& u_{a}=0 \text { must be hold. }
\end{aligned}
$$

Substituting (3) into (2e), we obtain that $v_{a}$ does not change with time, and recalling that $\left.v_{a}\right|_{t=0}=0$, we may write:

$$
v_{a}=0
$$

From (3) and (4), the barotropic component of perturbation wind is always zero. Then substituting (3) and (4) into (2d), $\phi_{a}$ is also independent of $r$. The condition $\left.\phi_{a}\right|_{r \rightarrow \infty}=0$ leads to:

$$
\phi_{a}=0
$$

Multiplying (2b) by $r / 1+\bar{\zeta}$, and carrying out a transformation of $\partial / r \partial r$, then subtracting (2c), it reduces to: 


$$
\frac{\partial}{\partial t}\left[\phi_{d}-\frac{\partial}{r \partial r}\left(\frac{r v_{d}}{1+\bar{\zeta}}\right)\right]=Q(r, t)
$$

By integrating (6) with respect to time, we obtain:

$$
\phi_{d}-\frac{\partial}{r \partial r}\left(\frac{r v_{d}}{1+\bar{\zeta}}\right)=\int_{0}^{t} Q(r, \tau) d \tau+\left[\phi_{d}-\frac{\partial}{r \partial r}\left(\frac{r v_{d}}{1+\bar{\zeta}}\right)\right]_{t=0},
$$

With the initial conditions $\left.\phi_{d}\right|_{t=0}=0$ and $\left.v_{d}\right|_{t=0}=0$, (7) can be simplified as follows:

$$
\phi_{d}=\frac{\partial}{r \partial r}\left(\frac{r v_{d}}{1+\bar{\zeta}}\right)+\int_{0}^{t} Q(r, \tau) d \tau
$$

Differentiating both sides of Eq. (2b) with respect to $t$ yields:

$$
\frac{\partial^{2} v_{d}}{\partial t^{2}}+(1+\bar{\zeta}) \frac{\partial u_{d}}{\partial t}=0
$$

and substitution of (2a), (9) reduces, after rearrangement, to:

$$
\frac{1}{1+\bar{\zeta}} \frac{\partial^{2} v_{d}}{\partial t^{2}}+\left(1+\frac{2 \bar{v}}{r}\right) v_{d}-\frac{\partial \phi_{d}}{\partial r}=0
$$

Differentiating (8) with respect to $r$, then substituting it into (10), we obtain the equation for $v_{d}$, i.e.,

$$
\frac{1}{1+\bar{\zeta}} \frac{\partial^{2} v_{d}}{\partial t^{2}}+\left(1+\frac{2 \bar{v}}{r}\right) v_{d}-\frac{\partial}{\partial r}\left[\frac{\partial}{r \partial r}\left(\frac{r v_{d}}{1+\bar{\zeta}}\right)\right]=\int_{0}^{t} \frac{\partial Q(r, \tau)}{\partial r} d \tau
$$

with the definition $\hat{v}=\frac{v_{d}}{1+\bar{\zeta}}$ we can rewrite (11) as follows:

$$
\frac{\partial^{2} \hat{v}}{\partial t^{2}}+\left(1+\bar{\zeta}+\frac{2 \bar{v}}{r}+\bar{\zeta} \frac{2 \bar{v}}{r}+\frac{1}{r^{2}}\right) \hat{v}-\frac{1}{r} \frac{\partial \hat{v}}{\partial r}-\frac{\partial^{2} \hat{v}}{\partial r^{2}}=\int_{0}^{t} \frac{\partial Q(r, \tau)}{\partial r} d \tau
$$


Eq. (12) governs the time and space variation of the perturbation $\hat{v}$ during the adjustment process of the initial balanced vortex to the heat forcing with the initial conditions: $\hat{v}_{i}^{0}=0$, $\hat{v}_{i}^{1}=\hat{v}_{i}^{0}-u_{i}^{0} \Delta t$, and the boundary conditions: for $r=0, \hat{v}_{1}^{n}=0$; for $r \rightarrow \infty, \hat{v}_{\infty}^{n}=0$,. The numerical solution, using centered space and time differences, for prescribed heat source will be presented in the next section. The nondimensional time step is $\Delta t=10^{-4}$, the radial grid size $\Delta r=10^{-3}$ and the simulation domain covers 10001 grids.

\section{SOLUTIONS WITH A SPECIFIED HEAT SINK}

To represent the initial balanced flow as an idealized but realistic tropical cyclone, the basic state tangential wind is given as follows:

$$
\bar{v}=a\left[2-e^{-b r}\left(b^{2} r^{2}+2 b r+2\right)\right] /(b r),
$$

where $a=4.0, b=40.0$. The corresponding relative vorticity is $\bar{\xi}=a b^{2} r e^{-b r}$. Figure 2a displays the radial profile of tangential wind of the basic state. It is evident from Fig.2a that for the initial balanced vortex, the tangential wind is zero at the center, and increases with $r$ up to the maximum (the dimensional value, $V_{\max }=62.4 \mathrm{~m} \mathrm{~s}^{-1}$ located at $r_{\max }=67.2 \mathrm{~km}$ ) then decreases with $r$, representing a realistic tropical cyclone wind structure. Furthermore, we include a specified rate of external heat sink term in (12) that can be separated into a spacedependent and a time-dependent part, i.e.,

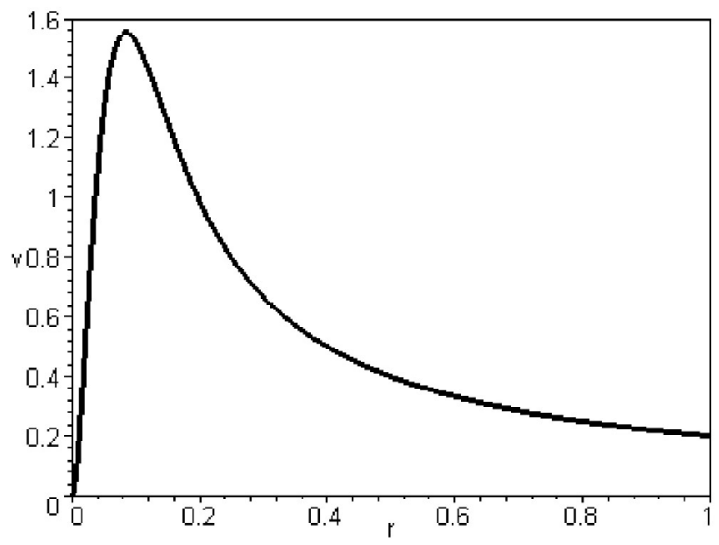

Fig. 2. Radial profile of tangential wind of the basic state vortex. The abscissa is the non-dimensional TC radius, $r=0.1$ represents $80 \mathrm{~km}$ and the tangential wind $v=1$ displays $40 \mathrm{~m} \mathrm{~s}^{-1}$. The maximum tangential wind $\left(62.4 \mathrm{~m} \mathrm{~s}^{-1}\right)$ of TC is located at $r=67.2 \mathrm{~km}$. 


$$
Q(r, t)=Q_{r}(r) Q_{t}(t)
$$

where

$$
Q_{t}(t)=\alpha^{2} t e^{-\alpha t}
$$

A smaller $\alpha$ corresponds to a slower heating or cooling rate but the total amount of forcing is independent of $\alpha$ since $\int_{0}^{\infty} \alpha^{2} t e^{-\alpha t} d t=1$. Here we use $\alpha=1$. The spatial distribution of the heat sink is given in the form of:

$$
Q_{r}(r)=C^{*}\{\operatorname{erf}[A(r-B)]-1\},
$$

where $C=4.0$, and $A=40.0$ representing the spatial variation of the heat sink, and the parameter $B$ indicates the location of the heat sink.

In this section, three experiments are set up by choosing different values of $\mathrm{B}$, i.e., $\mathrm{B}=0.14$; $\mathrm{B}=0.10 ; \mathrm{B}=0.17$ in TEST1, TEST2, and TEST3, respectively. The corresponding dimensional value of the strongest heat sink is about $-2.97 \mathrm{~K} \mathrm{hr}^{-1}$. The time- and spatial- variation of the heat sink for these three experiments are given in Figs.3, 6a and 7a., respectively. The heat sink increases from zero to its maximum magnitude (negative value) at $t=1.0$, then decreases to zero by $t=10.0$ (Fig.3a). Meanwhile, the maximum intensity of the heat sink is situated in the center region of the vortex, and it decreases with quickly beyond $r=0.1$, where the radius of basic state maximum wind is (see Figs.3b, 6a and 7a). This heat sink is selected to represent forcing during the late stage of strong convection after the storm reached its maximum intensity or a sudden cold advection from the environment.
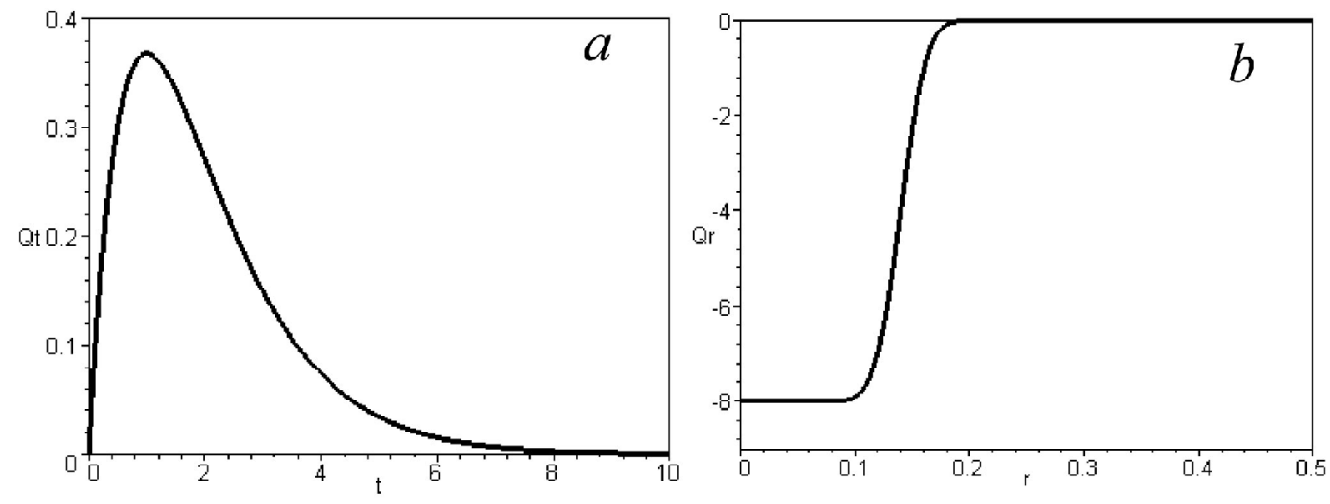

Fig. 3. (a) Temporal variation of the heat sink $Q_{t}(t)$ for TEST1-5, and (b) spatial variation of the heat sink $Q_{r}(r)$ for TEST1. The strongest heat sink is about $-2.9 \mathrm{~K} \mathrm{hr}^{-1}$ and $t=1$ equals to $5.56 \mathrm{hr}$. 
Following the experimental design and Eq. (12), we can calculate the vertical shear of the tangential wind perturbations $\left(v_{d}\right)$ in different time steps, and using (2b) and (8) to obtain the vertical shear of the radial wind perturbations $\left(u_{d}\right)$ and thickness $\left(\phi_{d}\right)$, and the perturbations at level 1 and level 3. Here, we only show the evolution of the disturbance at level 3. Figures $4 \mathrm{a}$ and $\mathrm{b}$ depict the time variation of the radial wind perturbations at level 3 of TEST1. It can be seen that, in response to the heat sink, the initially balanced vortex experiences an imbalance and starts to adjust between the wind and the geopotential field. The radial wind perturbations $\left(u_{3}\right)$ increases gradually outside RMW in the early stage. Later on, it decreases through the outward propagation of inertia gravity waves. After the inertia gravity wave propagates away, the vortex reaches a new balance with $u_{3}$ vanishing. The perturbation radial wind maximum is located at $r=0.15$, where the heat sink has the largest spatial variation (Fig. 3b). In other words, the radial profile of radial wind perturbation depends critically on the spatial distribution of the heat sink and can be identified from (2a) and (2c).

Figure $4 \mathrm{c}$ depicts the time evolution of the tangential wind perturbations $\left(v_{3}\right)$ during the adjustment process. Unlike the radial component $\left(u_{3}\right)$, tangential wind perturbation increases anticyclonically with time and reaches a quasi-steady state. Since the evolution of $v_{3}$ is not only associated with but also depends on the relative vorticity of the initial balanced vortex, the maximum of $v_{3}$ is located near the position where the basic state maximum wind was. In other words, the linear model with specified heat sink predicts that a maximum reduction of tangential wind at RMW will be induced. This result indicates that the resultant tangential wind will be in the form of a double-peak (similar to a concentric-eyewall structure in terms of tangential flow of an observed tropical cyclone) after a heat sink is imposed.

Figure $4 \mathrm{~d}$ depicts the time variation of the total tangential wind at level $3\left(V_{3}=\bar{v}+v_{3}\right)$. It is clear that the radial profile of the tangential wind displays a double-peak structure. In the beginning, the inner peak of the wind speed is larger than the outer one. Later on, the difference between the double peaks gradually diminishes, and finally the outer peak becomes greater than the inner one. The evolution of the tangential wind bears some resemblance to that of hurricane "Gilbert" (see Fig.1).

The adjustment process in the presence of a heat sink in a simulated tropical cyclone can be delineated in a schematic diagram shown by Fig. 5. In the beginning there is no heat sink, and the vortex maintains a gradient wind balance, i.e.,

$$
-\left(f+\frac{2 V_{0}}{r}\right) V_{0}=-\frac{\partial \Phi_{0}}{\partial r}
$$

where $V_{0}=\bar{v}, \Phi_{0}=\bar{\phi}$. In the presence of a heat sink, the thickness between $P_{1}$ and $P_{3}$ becomes thinner in the vicinity of the vortex center where the heat sink applies (from Eq.2c). This means the geopotential at level $3\left(P_{3}\right)$ will rise, i.e., $\phi_{3}>0$. Meanwhile, as there is no forcing away from the heat sink, the geopotential at level 3 is nearly unchanged far away, resulting in a modification of the radial distribution of the total geopotential height $\left(\Phi=\bar{\phi}+\phi_{3}\right)$ at level 3, i.e., $\partial \Phi / \partial r<\partial \Phi_{0} / \partial r$. Therefore, the pressure gradient force decreases and cannot be in balance with the sum of the Coriolis and the centrifugal forces. Consequently, there is an 

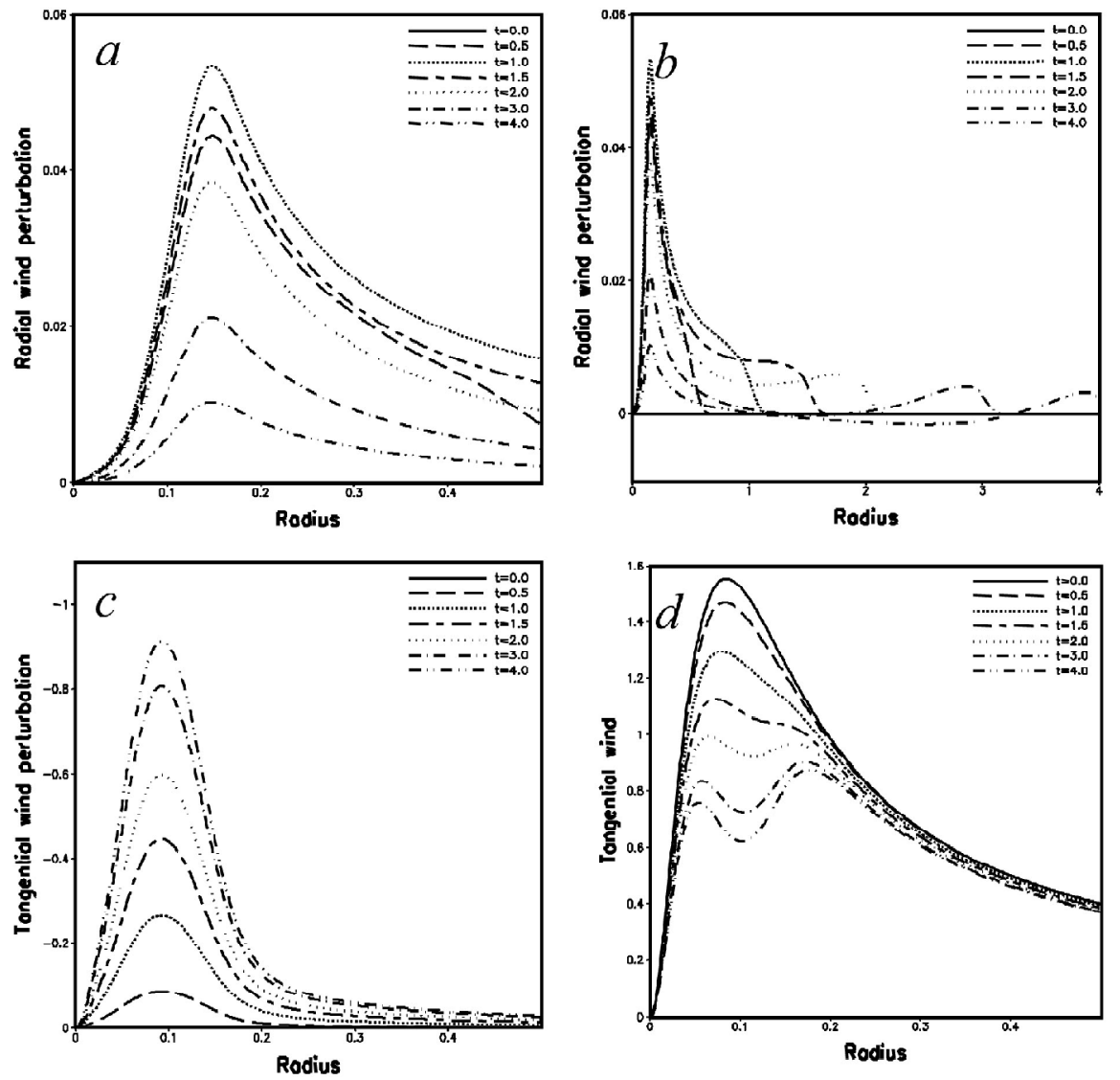

Fig. 4. Radial profiles of the level 3 perturbations of radial wind (a) in the inner core, (b) the outer region, (c) the tangential wind, and (d) total tangential wind at different time steps of TEST1. The abscissa is the non-dimensional TC radius, $r=0.1$ means $80 \mathrm{~km}$ and the non-dimensional wind speed $v=1.0$ displays $40 \mathrm{~m} \mathrm{~s}^{-1}$.

outgoing radial velocity perturbation $\left(u_{3}>0\right)$. From Eq. 8, we can see that $\partial \Phi_{3} / \partial r$ depends on the radial distribution of the heat sink. A greater $\left|\partial Q_{r} / \partial r\right|$ means a greater $\left|\partial \phi_{3} / \partial r\right|$. The perturbed radial flow due to the earth's rotation will induce an anticyclonic tangential wind perturbation that is in a direction opposite to the basic state tangential flow. The net result of this adjustment process is a decrease in the total tangential wind of the vortex.

From Eq. 2b, the radial distribution of the perturbation tangential wind depends on the multiplication of the radial outflow and the relative vorticity of the initial vortex. It is evident in Fig. 4, the maximum anticyclonic perturbation tangential wind is located near $r=0.1$ and the linear feedback to the tangential wind is at its largest. The induced anticyclonic tangential 

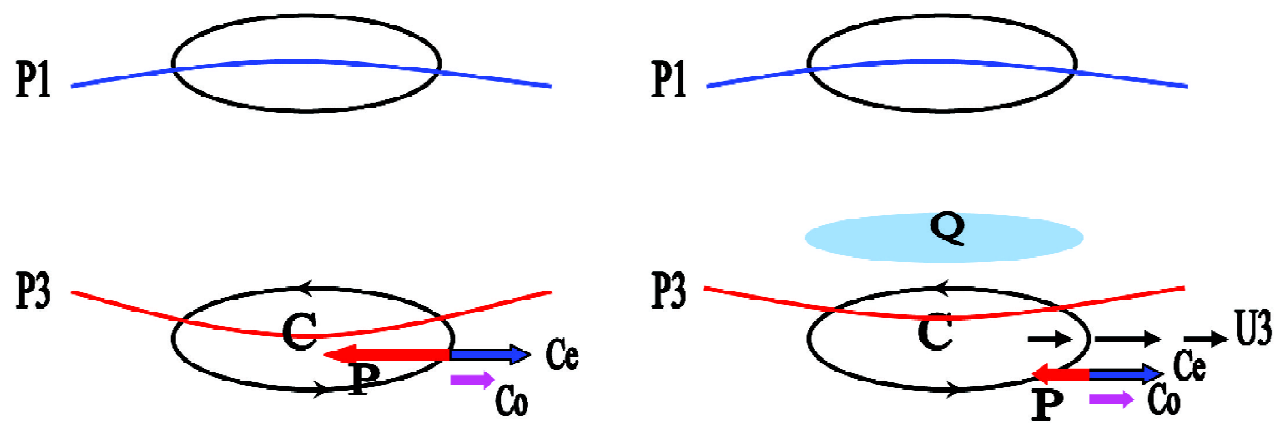

Fig. 5. Schematic diagram of the gradient adjustment process discussed in the paper. $U_{3}$ indicates radial outflow, $\mathrm{P}_{1}, \mathrm{P}_{3}$ refer to the upper- and lowerlevels, and $\mathrm{C}$ the low-level cyclone, $\mathrm{Q}$ the heat sink, $\mathrm{P}, \mathrm{Ce}$ and Co present pressure force, centrifugal force, and Coriolis force, respectively. The left panel refers to the initial state, and the right panel indicates the final adjusted state.

flow, when imposed on the basic cyclonic flow, will display a double-peak like wind profile. Since mid-level cooling can exist during the weakening stage of a strong storm, the aforementioned mechanism can play a role in the formation of concentric double eyewalls in a strong tropical cyclone, among other processes.

Figures 6 and 7 display the simulations of TEST2, and TEST3, respectively. The time variation of the perturbations of the radial wind (Figs. 6b, 7b) and the tangential wind (Figs. 6c, 7c) at level 3 of TEST2 are similar to that of TEST3. The comparison of the two experiments indicates that the location of the maximum radial wind perturbations agree roughly with that of the radial variation of the heat sink, indicating that the evolution of the radial wind perturbation is a result induced by the external heat sink imposed in the linear model. On the other hand, the maximum perturbation tangential wind is not located at the position of the largest radial variation of the heat sink. Instead, it is located closer to RMW, especially for the TEST3 case. The major difference among TEST1, TEST2, and TEST3 is the positional difference of the maximum perturbation tangential wind. This is due to the different radial distributions of the heat sink of these three cases. There are some differences, in terms of time evolution, during the formation of the double-peak structure. For TEST1, the tangential wind of the inner peak is larger than that of the outer one in the early stage and reversed in the later stage. For TEST2, the tangential wind of the inner peak decreases sharply and is always smaller than that of the outer peak during the whole period. This feature of TEST3 is the reverse of TEST2 such that the tangential wind of the outer peak is smaller than the inner one and is different from that of experiments TEST2 and TEST1. In summary, different cooling profiles can induce different 

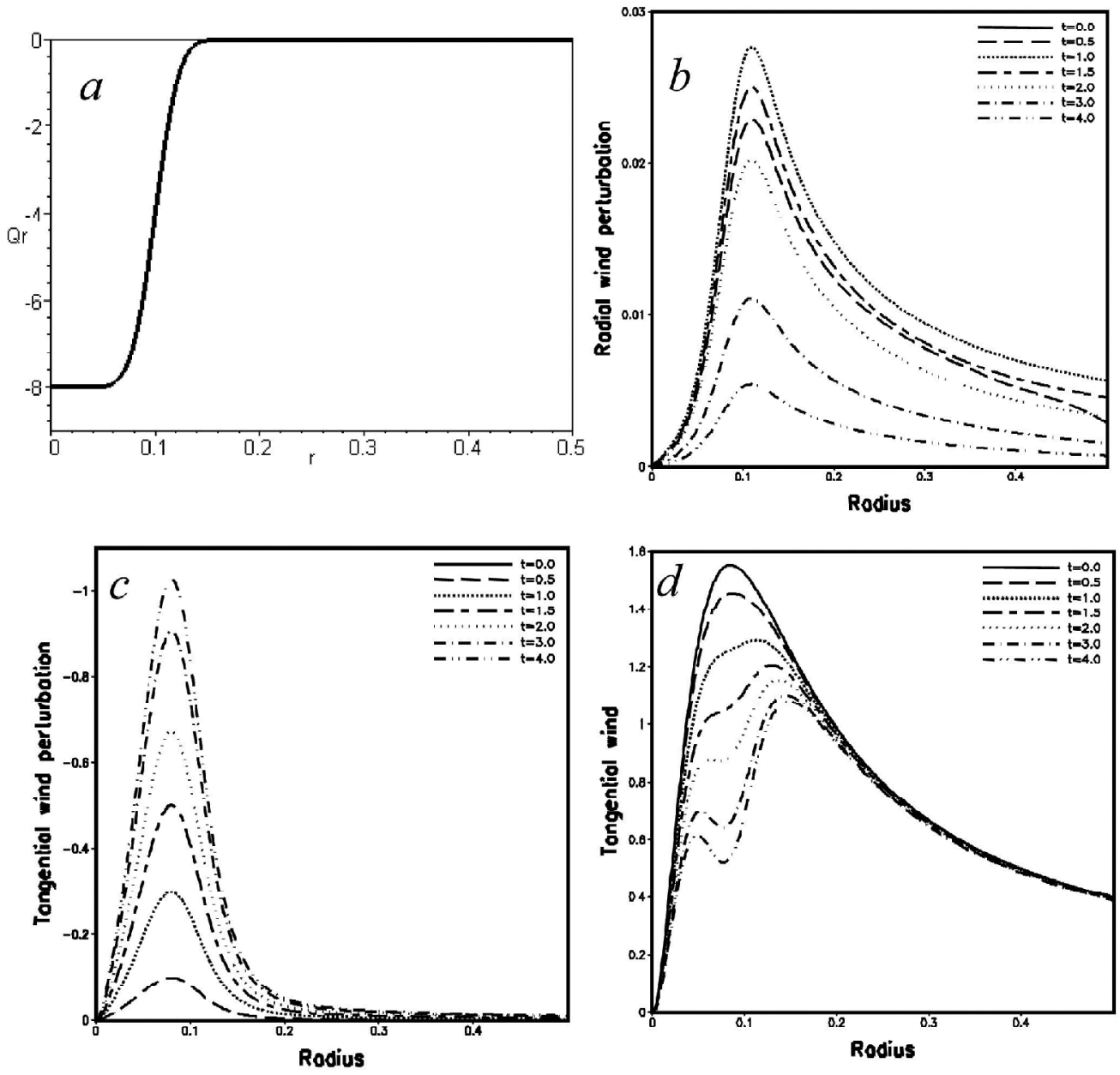

Fig. 6. The spatial variation of the heat sink $Q_{r}(r)$ (a), the radial profiles of the level 3 perturbations of radial wind (b), tangential wind (c), and total tangential wind (d) in different time steps of TEST2. The abscissa is the non-dimensional TC radius, $r=0.1$ means $80 \mathrm{~km}$ and the non-dimensional wind speed $v=1.0$ displays $40 \mathrm{~m} \mathrm{~s}^{-1}$.

perturbation winds at different locations. The induced tangential wind perturbations, when added to the original basic state tangential wind profile, exhibit a double-peak tangential wind structure that bears some resemblance to the concentric-eyewall feature commonly observed in intense tropical cyclones. 

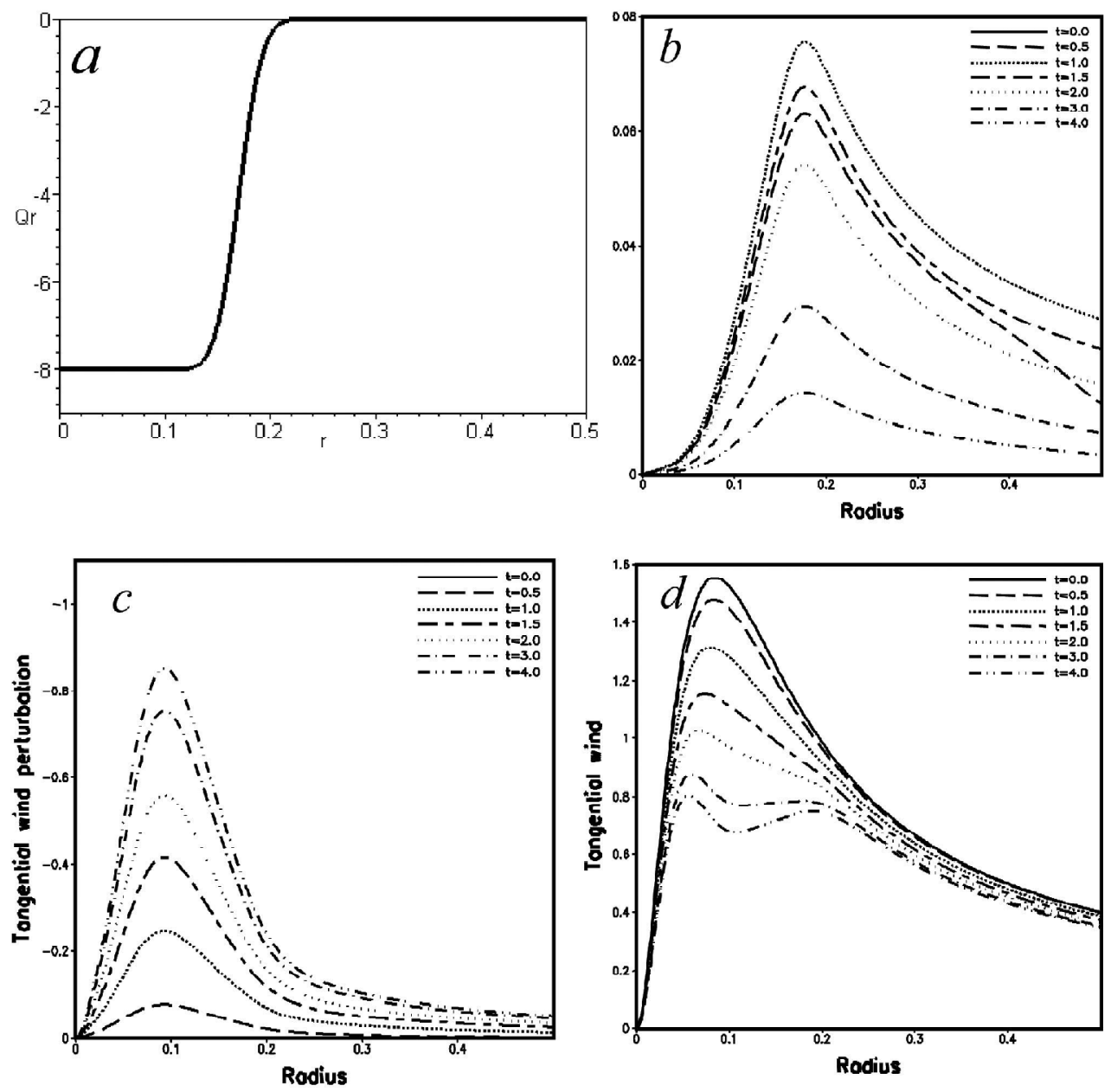

Fig. 7. The spatial variation of the heat sink $Q_{r}(r)$ (a), the radial profiles of the level 3 perturbations of radial wind (b), tangential wind (c), and total tangential wind (d) in different time steps of TEST3. The abscissa: the non-dimensional TC radius, $r=0.1$ means $80 \mathrm{~km}$ and the non-dimensional wind speed $v=1.0$ displays $40 \mathrm{~m} \mathrm{~s}^{-1}$.

\section{CONTRACTION OF THE OUTER EYEWALL}

Shapiro and Willoughby (1982) and Willoughby et al. (1982) used an axisymmetric model (SW model) to discuss secondary circulation induced by point heat source in balanced vortices. 
For a heat source near RMW, a sharp peak of the tangential wind tendency occurred just inside of RMW. This perturbation wind response results in a propagation of RMW toward the center. This provides a plausible explanation for the observed contraction of the eyewall in intensifying hurricanes. For comparison with their results, we conduct the fourth test (here after, TEST4) in this study. The basic state tangential wind is the same as TEST1; however, the heat source in (12) is decomposed into space-dependent and time-dependent parts, respectively, i.e., $Q(r, t)=Q_{r}(r) Q_{t}(t)$, where the time-variation is the same as that of TEST1. Nevertheless, the spatial distribution of the heat source is given in the form of:

$$
Q_{r}(r)=\frac{20}{\sqrt{2 \pi \sigma}} e^{\left[-\frac{1}{2}\left(\frac{r-\bar{r}}{\sigma}\right)^{2}\right]},
$$

where $\sigma=0.01$, and $\bar{r}=0.044$ displays the location of the maximum heating and is given in Fig. 8a. In this case, the maximum heating $\left(2.97 \mathrm{~K} \mathrm{hr}^{-1}\right)$ is set at $r=35.2 \mathrm{~km}$.

Time variation of the radial wind perturbation at level 3 of TEST4 is given in Fig. 8b. It can be seen that the outflow $\left(u_{3}>0\right)$ lay inside of the maximum heating $(r=0.044$, the dimensional scale $35.2 \mathrm{~km}$ ) and the inflow located just outside the maximum heating. In other words, the spatial distribution of the heat sink has noticeable effect on the radial wind perturbations. The evolution of the tangential wind perturbations $\left(v_{3}\right)$ during the adjustment process is given in Fig. 8c. Consistent with the change of the radial wind perturbation, the tangential wind perturbations are negative inside the point of maximum heating $(r=0.044)$ and positive outside of it. The resultant total tangential wind $\left(V_{3}=\bar{v}+v_{3}\right)$ at level 3 (Fig. 8d) indicates, in response to the heating, the vortex intensifies with its RMW moving inward. This result is consistent with that of Shapiro and Willoughby (1982) and Willoughby et al. (1982).

The fifth experiment (TEST5) in this study is designed to demonstrate the possible contraction of the outer peak for a concentric-eyewall structure. Based upon TEST1, a heat source is added after the double-peak structure has formed at the time $t=2.0$. The spatial distribution of the heat source is given in the form:

$$
Q_{r}(r)=\frac{20}{\sqrt{2 \pi \sigma}} e^{\left[-\frac{1}{2}\left(\frac{r-\bar{r}}{\sigma}\right)^{2}\right]},
$$

where $\sigma=0.01$, and $\bar{r}=0.124$ displays the location of the maximum heating (Fig.9a). Time variation of the heating is the same as that of TEST1. The evolution of the radial wind perturbations at level 3 is depicted in Fig. 9b. It can be seen that from $t=0.0$ to $t=2.0$, during the cooling period, the outflow increases between $t=0.0-1.0$ and then decreases afterwards. Starting $t=2.0$ the heating begins to force the vortex. The outflow $\left(u_{3}>0\right)$ is located inside the maximum heat source, accompanied by an inflow outside this radius $(r=0.124)$. Associated with the radial wind perturbations, the total tangential wind at level 3 (Fig. 9c) decreases after the vortex is forced by the cooling initially. At $t=2.0$, the radial profile of the tangential 
wind displays double-peak structure. After that, the heat source located at the outer eyewall starts working, and induces the contraction of the outer eyewall. By comparing the radial distribution of the tangential wind at $t=3.0$ and $t=5.0$, the outer eyewall strengthens and contracts while the inner eyewall shows noticeable weakening. The evolution of the tangential wind simulated in this experiment is very similar to that observed in hurricane Gilbert.
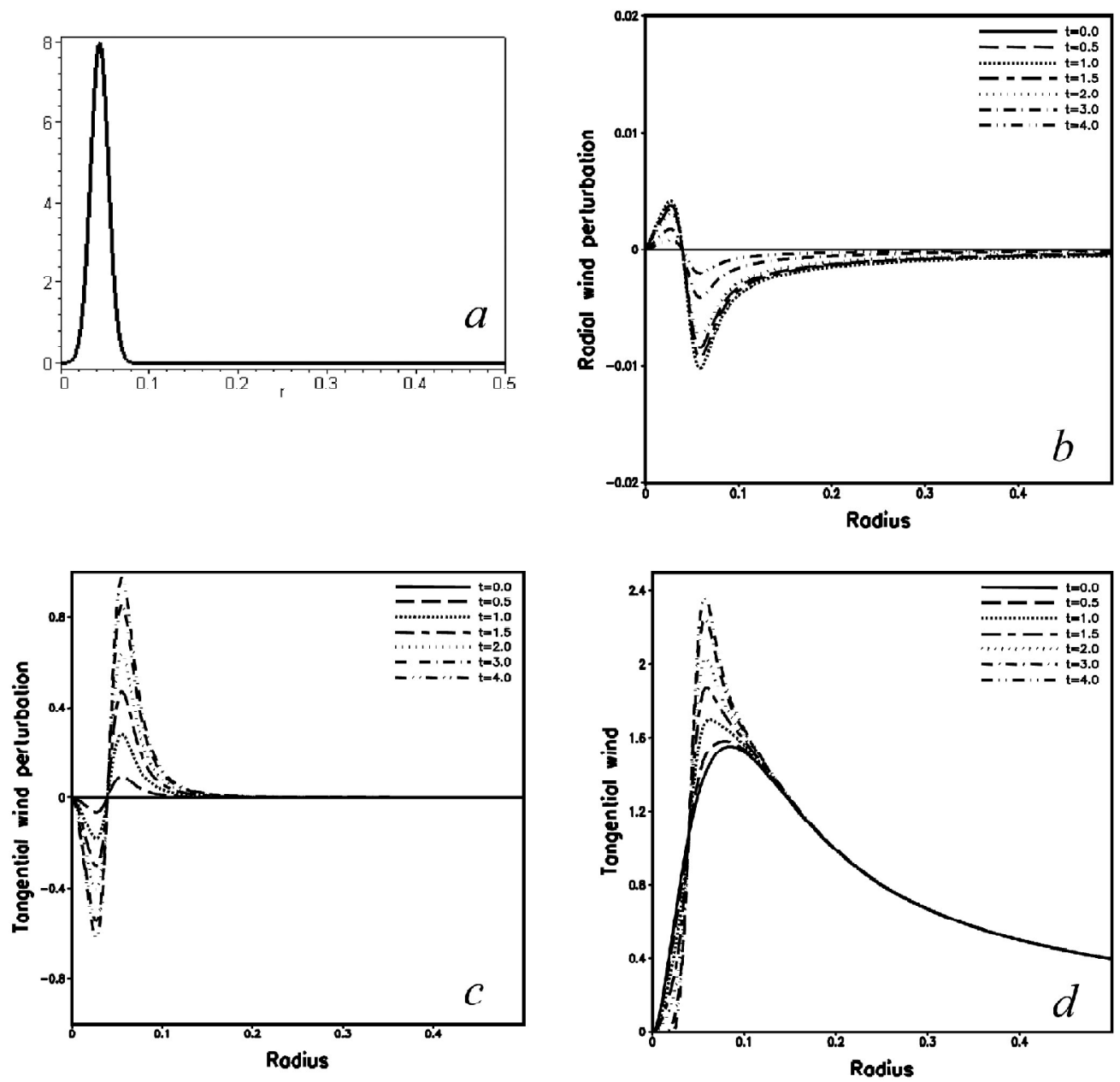

Fig. 8. The spatial variation of the warm source $Q_{r}(r)$ (a), the radial profiles of the level 3 perturbations of radial wind (b), tangential wind (c), and total tangential wind (d) in different time steps of TEST4. The abscissa: the non-dimensional TC radius, $r=0.1$ means $80 \mathrm{~km}$ and the non-dimensional wind speed $v=1.0$ displays $40 \mathrm{~m} \mathrm{~s}^{-1}$. 

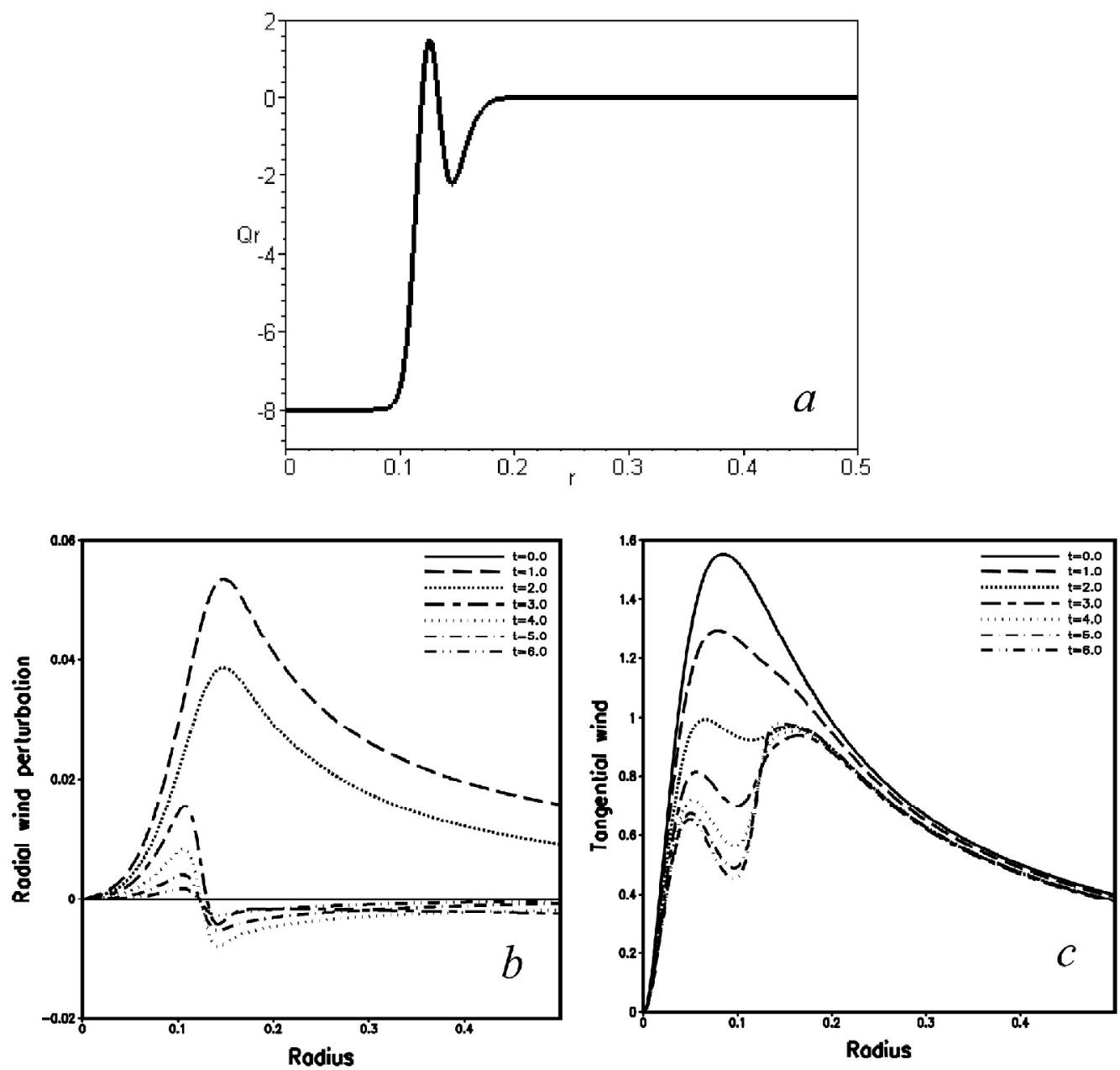

Fig. 9. The spatial variation of the warm source $Q_{r}(r)$ (a), the radial profiles of the level 3 perturbations of radial wind (b), and total tangential wind (c) in different time steps of TEST5. The abscissa: the non-dimensional TC radius, $r=0.1$ means $80 \mathrm{~km}$ and the non-dimensional wind speed $v=1.0$ displays $40 \mathrm{~m} \mathrm{~s}^{-1}$.

\section{CONCLUDING REMARKS}

In the context of a linear two-layer axisymmetric model, the gradient adjustment process of the initial balanced vortex to a prescribed heating/cooling source is discussed in this paper. The cooling effect represents phenomena such as re-evaporation of precipitation and/or cold advection from the environment flow in the decaying period of strong tropical cyclones. With 
the heat sink, a perturbation tangential wind is induced in a gradient adjustment that is opposite to the basic vortex and with its maximum amplitude located near RMW of the basic vortex. With this perturbation tangential wind, a cyclone-like vortex displays a double-peak in its tangential wind distribution similar to that of concentric eyewalls in tropical cyclones. It is demonstrated in this simple model simulation study that with gradient adjustment of the initially balanced vortex in response to a specified heat sink, the formation of the concentric eyewalls can be realized. By including an additional heat source at a stage after the outer eyewall formation, this model again successfully simulates the inward contraction of the outer eyewall and the accompanying weakened inner eyewall. The results presented in this paper resemble, in a profound way, the formation of a double-peak tangential wind feature in a tropical cyclone, and furthermore, the replacement of the inner peak of the tangential wind by the outer one. Disregarding the simple formulation of the linear two-layer model, it has been demonstrated in this paper that the temporal and spatial distribution of heating/cooling induced either by external forcing or internally by the convective activity have a fundamental role to,play in the structural evolution of a tropical cyclone.

As noted by Nong and Emanuel (2003), based on work using two axisymmetric models, i.e., a simple two-layer balanced dynamic model combined with a cloud-resolving non-hydrostatic model, a real secondary eyewall may result from a finite-amplitude WISHE (wind-induced surface heat exchange) instability, triggered by external forcing, such as the interaction of a tropical cyclone with baroclinic eddies, topography, or local perturbations in sea surface temperature. It is demonstrated in the current work that with external forcing (small disturbances in heating), the model successfully simulates the formation of a secondary eyewall, and completes the replacement cycle. From this study, it is suggested that more works could be conducted under this simple but computationally efficient axisymmetric framework to investigate intensity changes of tropical cyclones caused by the WISHE instability as proposed by Nong and Emanuel (2003).

Acknowledgements This work was sponsored by the Postdoctoral fellowship of the first author at National Taiwan University granted by NSC of Taiwan (NSC 91-2111-M-002-023AP4, NSC 92-2816-M-002-0003-6, NSC 92-2111-M-002-020-AP2) and the National Natural Science Foundation of China under Grant 40205009, 40175005 and 40075011; the Observation and Prediction scheme of Landfall Tropical Cyclone Disaster (2001P1A20026). The authors are very grateful to Drs. Zhang Dalin, University of Maryland, Dr. Xue Min, University of Oklahoma, Dr. Tan Zhemin, Nanjing University, and Dr. Chung-Hsin Sui, National Central University for their beneficial discussions.

\section{REFERENCES}

Black, M. L., and H. E. Willoughby, 1992: The concentric eyewall cycle of hurricane Gilbert. Mon. Wea. Rev., 120, 947-957.

Chen, S. M., 1987: Preliminary analysis on the structure and intensity of concentric double eye tropical cyclones. Adv. Atmos. Sci., 4, 113-118. 
Kuo, H., C., L. Y., Lin, C. P., Chang, and R. T., Williams, 2004: The formation of concentric vorticity structures in typhoons. J. Atmos. Sci., 61, 2722-2734.

Montgomery, M. T., and R. J. Kallenbach, 1997: A theory for vortex Rossby-waves and its application to spriral bands and intensity changes in hurricanes. Q. J. R. Meteor. Soc., 123, 435-465.

Nong, S., and K. A. Emanuel, 2003: A numerical study of the genesis of concentric eyewalls in hurricane. Q.J. R. Meteor. Soc., 129, 3323-3338.

Schubert, W. H., J. J. Hack, P. L. Silva Dias, and S. R. Fulton, 1980: Geostrophic adjustment in an axisymmetric vortex. J. Atmos. Sci., 37, 1464-1483.

Shapiro, L. J., and H. E. Wiloughby, 1982: The response of balanced hurricanes to local sources of heat and momentum. J. Atmos. Sci., 39, 378-394.

Willoughby, H. E., J. A. Clos, and M. G. Shoreibah, 1982: Concentric eye walls, secondary wind maxima, and the evolution of the hurricane vortex. J. Atmos. Sci., 39, 395-411.

Willoughby, H. E., H. L. Jin, Stephen J. Lord, and Jacqueline M. Piotrowicz, 1984: Hurricane structure and evolution as simulated by an axisymmetric non-hydrostatic numerical model. J. Atmos. Sci., 41, 1169-1186.

Willoughby, H. E., 1988: The dynamics of the tropical cyclone core. Aust. Met. Mag., 36, 183-191.

Willoughby, H. E., J. M. Masters, and C. W. Landsea, 1989: A record minimum sea level pressure observed in Hurricane Gilbert. Mon. Wea. Rev., 117, 2824-2828. 